\title{
Hydraulic performance evaluation of a micro-turbine with counter rotating runners by experimental investigation and numerical simulation
}

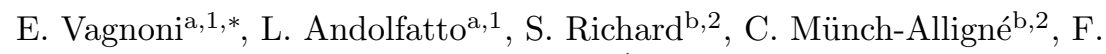 \\ Avellan ${ }^{\mathrm{a}, 1}$ \\ ${ }^{a}$ Av. du Cour 33 bis, 1004 Lausanne, Switzerland \\ ${ }^{b}$ Route du Rawyl 47, 1950 Sion, Switzerland
}

\begin{abstract}
The development of energy recovery systems on existing water utility networks is a new challenge for increasing the sustainability of electricity generation mix. The concept of the new micro-turbine with counter rotating runners braces this goal by allowing the recovery of the energy which is spoiled in pressure reducing valves in many water supply networks. The hydraulic performances of the micro-turbine are evaluated through an analysis on the angular momentum balance applied to the investigated cross-sections of the machine. For this analysis, an experimental campaign involving 2D Laser Doppler Velocimetry (LDV) is performed to measure the velocity field of the flow at the inlet and outlet sections of the machine. Since the optical access to perform LDV measurements is blocked close to the hub of the runners, a further analysis on the mass balance and numerical simulations are performed to complete the velocity profiles and to evaluate the machine performance. The combination of numerical and experimental methods grants a full study on the operating condition of the micro-turbine and strengthens the results achieved. The results back up the best operating condition of the machine for different discharges and support
\end{abstract}

\footnotetext{
* Corresponding author

Email addresses: elena.vagnoni@epfl.ch (E. Vagnoni), loic.andolfatto@epfl.ch (L. Andolfatto), sylvain.richard@hevs.ch (S. Richard), cecile.muench@hevs.ch (C. Münch-Alligné), francois.avellan@epfl.ch (F. Avellan)

${ }^{1}$ Laboratory for Hydraulic Machines, EPFL

${ }^{2}$ HES-SO Valais, Haute école spécialisée de Suisse occidentale
} 
further improvements of the runner blades shape.

Keywords: Small hydropower, micro-turbine with counter rotating runners, 2D LDV, CFD, energy production, efficiency

\section{Introduction}

Hydropower is the most exploited renewable energy at a global scale. In Switzerland it accounts for 36 TWh of average energy production and this value is expected to increase to $38 \mathrm{TWh}$ in 2050 . This increase stems by the improve5 ment of the efficiency of the machine in large hydropower plants by 2 TWh and by the development of mainly small hydropower plants by 1 TWh 1 .

Current research is focusing on the increase of the sustainability of small hydroelectricity generation by limiting the environmental impact and economical investments [2, [3] [4].

New turbines are developed for small hydropower plants since the conventional turbine are often not cost-effective due to the required high installations costs [5] 6. The concept of the new micro-turbine with counter rotating runners, illustrated in Fig. 1. braces this challenge by recovering energy which is spoiled in pressure reducing valves in existing water networks. The compact 15 axial architecture ensures a lean in-line installation on existing facilities. Variable rotational speed of the runners allows coping with the consumer-driven wide range of discharge usually experienced on water utility networks 7 . The development of the micro-turbine is focused on the study of one-stage design featuring two counter rotating runners to increase the flexibility of the machine operation. Stackable stages of counter-rotating runners enable to match the local pressure condition of the drinking water network 8] 9].

One challenge of this technology is to obtain the best hydraulic performance for a set of discharges depending on the water demand. Flow analysis allows evaluating the hydraulic performance of the turbine without being subject to distortion from mechanical losses. The standard measurements on the machine performance include also the mechanical power losses related to the mechanical 
components of the facility which are not related to the micro-turbine itself [8]. The solution of the angular momentum balance equation gives the hydraulic energy transferred by the fluid to the machine.

30 This paper presents an original method to estimate the hydraulic performance of the micro-turbine computed by solving the angular momentum balance equation. To solve this equation the velocity profiles of the flow at the inlet and outlet section of the micro-turbine must be measured.

Non-intrusive optical measurement such as LDV and Particle Image Velocimetry (PIV) are experimental techniques prominently developed in the past few years. These techniques fit well in turbomachinery applications as past studies have proved [10. As investigated precise results by these laser and optical measurement techniques are often difficult to achieve since parameters such as the refraction index of both the material interface and the flow, laser alignment, 40 seeding and machine geometry are fundamental to obtain accurate results [11. Successful set-ups were developed in the past to avoid optical distortion for the laser measurements 12, 13, 14, 15, [16.

LDV measurements are performed to investigate the velocity field of the flow in the micro-turbine. The laser beam reflection on the wall prevents the measurements close to the hub of the runners. To complete the velocity profiles a study on the mass balance equation and numerical simulations are carried out.

The power transfer in the turbine, including the power and efficiency definitions and the solution of the angular momentum balance equation, is described in Sec. 2. The experimental set-up and the numerical simulations are presented in Sec. 3, The results are described and discussed in Sec. 4.

\section{Power transfer in the turbine}

\subsection{Power and efficiency definitions}

The power and efficiency flow through a turbine is illustrated in Fig. 2. The specific energy available $E$ and the hydraulic power $P_{h}$ of a horizontal 


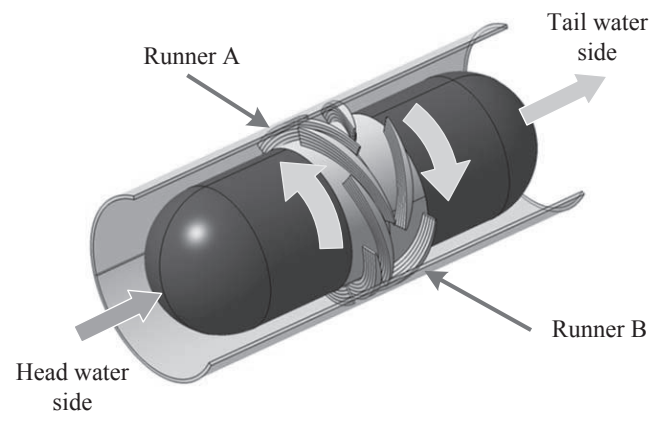

Fig. 1: Schematic representation of the axial micro-turbine with counter rotating runners.

turbine with a constant diameter are defined as follows:

$$
\begin{gathered}
E=g H_{\mathrm{I}}-g H_{\overline{\mathrm{I}}}=\frac{p_{\mathrm{I}}-p_{\overline{\mathrm{I}}}}{\rho} \\
P_{h}=\rho \cdot Q \cdot E
\end{gathered}
$$

In equation 1 , $p_{\mathrm{I}}$ and $p_{\overline{\mathrm{I}}}$ are the high pressure and low pressure side, respectively and $\rho$ is the density of water. In equation $2, Q$ is the discharge of the power unit.

The supplied power $P$ is defined as:

$$
P=\omega_{\mathrm{A}} \cdot T_{\mathrm{A}}+\omega_{\mathrm{B}} \cdot T_{\mathrm{B}}
$$

where $\omega_{\mathrm{A}}$ and $\omega_{\mathrm{B}}$ are the rotational speed of the first and second runners, respectively and $T_{\mathrm{A}}$ and $T_{\mathrm{B}}$ the measured torque.

By measuring the hydraulic power $P_{h}$ and the supplied power $P$, the efficiency $\eta$ is computed by obeying equation 4 .

$$
\eta=\frac{P}{P_{h}}
$$

Equation 4 takes into account the eventual losses of energy due to the mechanical losses through the mechanical components of the machine. To evaluate 55 the hydraulic performance of the turbine, the hydraulic efficiency $\eta_{h}$ must be investigated. 


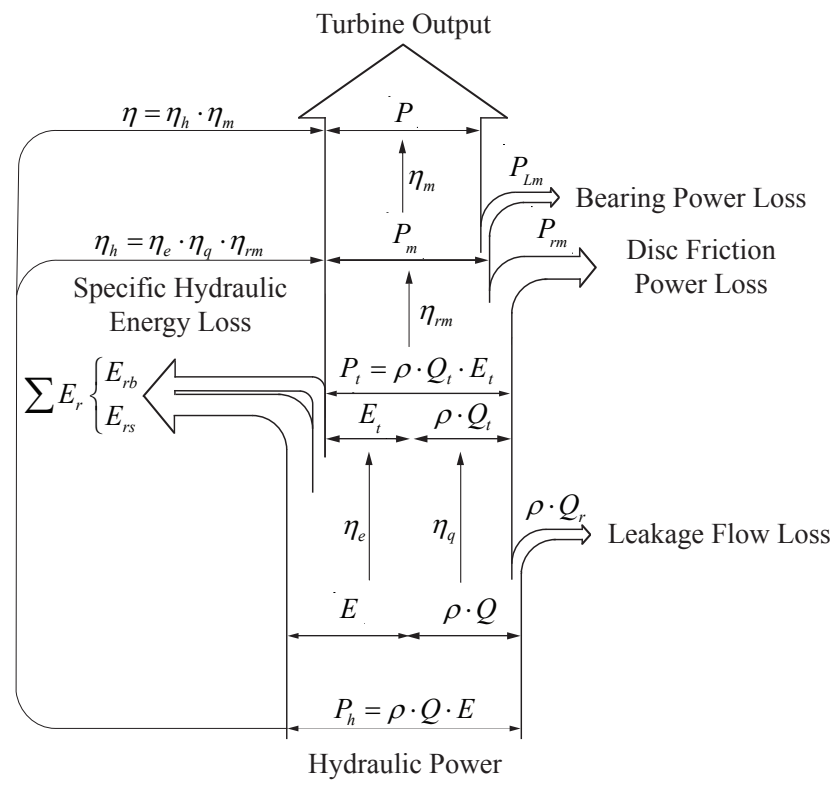

Fig. 2: Power flow through a hydraulic turbine 1].

By following the power flow illustrated in Fig. 2, the transformed power $P_{t}$ and the hydraulic power $P_{h}$ are defined as:

$$
\begin{gathered}
P_{t}=P_{m}+P_{r m} \\
P_{h}=P_{t}+P_{r e}+P_{r q}=P_{m}+P_{r m}+P_{r e}+P_{r q}
\end{gathered}
$$

where $P_{m}$ is the mechanical power, $P_{r m}$ are the power losses due to disc friction which are negligible for an axial turbine, $P_{r e}=\rho \cdot Q \cdot \sum E_{r}$ are the power losses due to the specific hydraulic energy losses are and $P_{r q}=\rho \cdot Q_{r} \cdot E$ are the power 60 losses due to the leakage flow losses.

This leads to compute the hydraulic efficiency as follows:

$$
\eta_{h}=\frac{P_{m}}{P_{h}}
$$

By replacing equation 7 in equation 6

$$
1=\eta_{h}+e_{r}+q_{r}
$$


where $e_{r}$ and $q_{r}$ are the losses factors due to the specific hydraulic energy losses and to the leakage flow losses. These losses factors can be rewritten as follows:

$$
e_{r}=1-\frac{E_{t}}{E} \quad q_{r}=1-\frac{Q_{t}}{Q}
$$

where $E_{t}$ and $Q_{t}$ are the transformed specific energy and the part of discharge which goes through the runners, respectively.

It is indeed necessary to investigate the transformed specific energy and discharge. To compute the transformed specific energy corresponding to the specific energy which is transferred by the flow to the machine without taking into account the mechanical losses due to the bearings, the angular momentum balance equation is solved both numerically and experimentally. The transformed discharge is computed by applying the mass balance equation on the runner radial section.

2.2. Solution of the angular momentum balance equation to compute the hydraulic performance

The angular momentum balance equation applied at each runner, defined in equation 10, yields to the transformed specific energy $E_{\mathrm{t}}$ from the flow to the runners with the hypothesis of inviscid fluid.

$$
\begin{aligned}
& E_{\mathrm{t}}=E_{\mathrm{t}, \mathrm{A}}+E_{\mathrm{t}, \mathrm{B}}=\frac{1}{Q} \int_{A_{1}^{\mathrm{A}} \cup A_{\overline{\mathrm{A}}}^{\mathrm{A}}} \vec{C} \cdot \vec{U} \vec{C} \cdot \vec{n} \mathrm{~d} A+\int_{A_{1}^{\mathrm{B}} \cup A_{\overline{1}}^{\mathrm{B}}} \vec{C} \cdot \vec{U} \vec{C} \cdot \vec{n} \mathrm{~d} A= \\
& =\frac{2 \pi}{Q} \int_{R_{\mathrm{h}}}^{R_{\mathrm{s}}} C_{\mathrm{u} 1 \mathrm{~A}}(r) C_{\mathrm{m} 1 \mathrm{~A}}(r) \omega_{\mathrm{A}} r^{2} \mathrm{~d} r+\frac{2 \pi}{Q} \int_{R_{\mathrm{h}}}^{R_{\mathrm{s}}} C_{\mathrm{u} \overline{1} \mathrm{~A}}(r) C_{\mathrm{m} \overline{1} \mathrm{~A}}(r) \omega_{\mathrm{A}} r^{2} \mathrm{~d} r+ \\
& \quad+\frac{2 \pi}{Q} \int_{R_{\mathrm{h}}}^{R_{\mathrm{s}}} C_{\mathrm{u} 1 \mathrm{~B}}(r) C_{\mathrm{m} 1 \mathrm{~B}}(r) \omega_{\mathrm{B}} r^{2} \mathrm{~d} r+\frac{2 \pi}{Q} \int_{R_{\mathrm{h}}}^{R_{\mathrm{s}}} C_{\mathrm{u} \overline{1} \mathrm{~B}}(r) C_{\mathrm{m} \overline{\mathrm{B}}}(r) \omega_{\mathrm{B}} r^{2} \mathrm{~d} r
\end{aligned}
$$

In equation $10 \vec{U}$ is the peripheral velocity computed as $U(r)=\omega \cdot r, C_{\mathrm{m}}(r)$ and $C_{\mathrm{u}}(r)$ are the meridional and tangential velocities, respectively, at the inlet 1 and at the outlet $\overline{1}$ sections of the runner $\mathrm{A}$ and $\mathrm{B}$.

75 To solve this equation the meridional and tangential velocity profiles of the flow at the inlet and outlet sections of the machine must be investigated. This justified the need of conducing an experimental campaign involving non-intrusive optical measurements. 


\section{Methodology}

80 3.1. Experimental set-up

An experimental investigation is conducted in the on scale model of the micro-turbine, shown in Fig. 3. The micro-turbine features a single stage axial turbine with two counter rotating runners; the first runner with five blades and the second runner with seven blades. The relevant geometrical parameters are

The micro-turbine is installed in an elbow pipe and a honeycomb is located upstream the inlet section to minimize the non-uniformity and large scale turbulence at the inlet of the first runner. An external pump with a maximum power of $7.5 \mathrm{~kW}$ feeds the facility taking water from an atmospheric pressure water tank and it is controlling the head for different machine operations.

The facility is equipped with two differential pressure transducers (Emerson, Rosemount 3051S) measuring the difference of the static pressure between the headwater and the tailwater sections as shown in Fig. 3. They have a precision of \pm 2.5 mbar. An electromagnetic flowmeter (Yokogawa, ADMAG SE AE205MG) located upstream the machine measures the discharge with $\pm 0.5 \%$ accuracy. The measured discharge is corrected by a loss coefficient due to the leakages through the seals. Each runner shaft is equipped with a strain gauges torquemeter (HBM, T22) for measuring the runner torque with $\pm 0.5 \%$ precision.

LDV measurements are performed in the inlet, inter-runner and outlet section of the machine. An external trigger from the burst spectrum analyzer (BSA) is used to set off the static pressure measurements, to assure the synchronization with the LDV measurement device. The runners are installed in a transparent casing. As illustrated in Fig. 3. the casing is equipped with three optical circular windows made out of N-BK7 glass. The thickness of the glass windows is $6 \mathrm{~mm}$ with a $32.5 \mathrm{~nm}$ flatness. This is designed to obtain a casing segment which avoids distortion and refraction of the laser beam and enlargement of the control volume during LDV measurements.

The experimental set-up for performing LDV measurements and the chain of 


\begin{tabular}{c|cc}
\multicolumn{3}{c}{ Tab. 1: Runners geometry } \\
Parameters & Symbol & Value \\
\hline Inner casing radius & $R_{1}$ & $50.3 \mathrm{~mm}$ \\
Shroud radius & $R_{\mathrm{S}}$ & $49.95 \mathrm{~mm}$ \\
Hub radius & $R_{\mathrm{h}}$ & $40 \mathrm{~mm}$ \\
Blade tip radial clearance & $\mathrm{s}$ & $0.35 \mathrm{~mm}$
\end{tabular}

acquisition is schematized in Fig. 4. The LDV system is a Dantec FlowExplorer compact LDA with two laser beams at $660 \mathrm{~nm}$ and $775 \mathrm{~nm}$. It is composed of a $300 \mathrm{~mW}$ solid state laser source, a beam splitter and an optical probe of $300 \mathrm{~mm}$ focal length. The seeding particles are $10 \mu \mathrm{m}$ diameter hollow glass spheres with a density that is almost the same as the one of water. It is therefore assumed that the particles behave as the liquid flow. A remotely controlled traversing system is used for the radial displacement of the laser. LDV measurements are performed to measure the average meridional component (along the $x$-direction) and the tangential component (along the $y$-direction) of the absolute flow velocity at different positions across the two runners. The origin of the Cartesian coordinates system is located between the two runners on the axis of the machine as shown in Fig. 4. The point for which the location is controlled during the measurement is the center of the measurement volume.

The measurements are performed at three positions along the $x$-axis, as indicated in Fig. 4 at the inlet of the first runner (Inlet section), between the two runners (Inter-runners section) and at the outlet of the machine (Outlet section). For each axial position, $8 \mathrm{~mm}$ on the $z$-axis are investigated with $0.1 \mathrm{~mm}$ spatial resolution. Further radial positions closer to the hub could not be investigated because of the reflection of the laser beams on the hub wall. For each axial and radial position, 20'000 samples are recorded and the maximum measurement duration is set to $120 \mathrm{~s}$. 


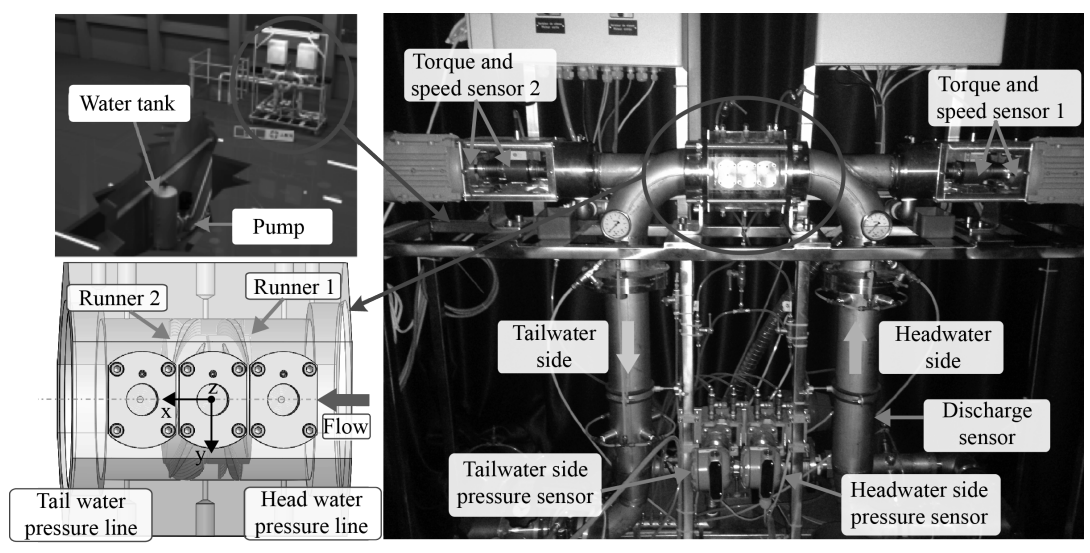

Fig. 3: Side view of the test facility.

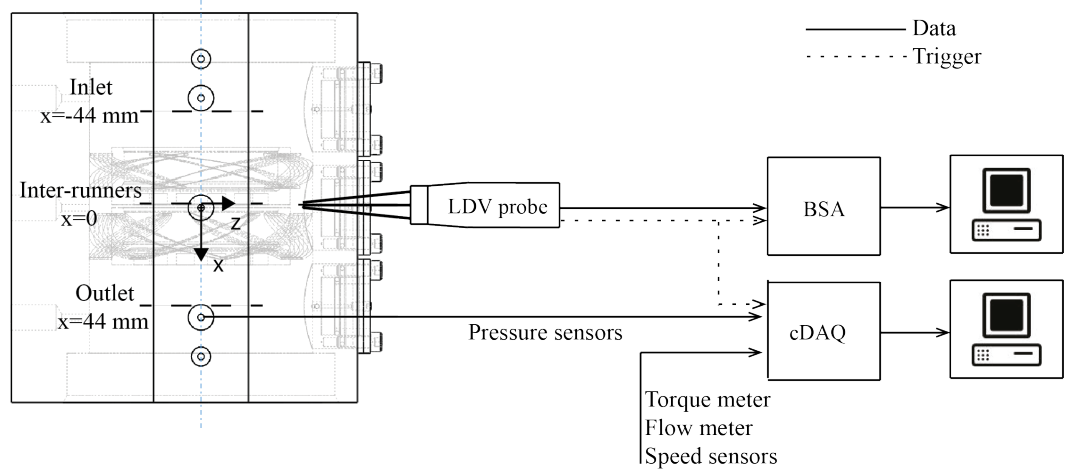

Fig. 4: Top view of the micro-turbine with counter rotating runners and measurements chain.

\subsection{Numerical simulations}

Steady flow numerical simulations of the flow in the micro-turbine are performed with the commercial ANSYS CFX 16.2 software based on the finite volume method. The Reynolds-Averaged Navier-Stokes equations are solved in their conservative form and are closed-formed using the shear stress transport (SST) two-equation turbulence model [17]. This turbulence model uses the komega model [18] close to the walls and the k-epsilon model [19] elsewhere. The set of equations are discretized according to an advection scheme with a specified blend factor corresponding to a second order in space. The convergence 
criteria based on the maximum values of the RMS is set to $1 \times 10^{-10}$.

In this study, the computing domain includes a straight pipe for the inlet, the turbine with the two runners and the second elbow with the shaft, as illustrated in Fig. 5. The stabilizing effect of the honey comb is sufficiently high to assume an axial flow at the inlet of the turbine. The blade tip radial clearance between the runner blades and the duct is considered. Simulations with and without tip radial clearance are performed. A structured mesh is used for the discretization, as illustrated in Fig. 6 and Fig. 7, with a maximum number of 3'946'863 nodes with the tip radial clearance and 3'441'731 nodes without the tip radial clearance; the different characteristics are given in Tab. 2, The computing domain is made of six parts, defined in Tab. 2, connected by five interfaces. There are two stationary parts and four rotating parts. For all the simulations, the interfaces between the stationary parts and the runners are frozen rotor conditions. Between each runner and the tip radial clearance sections, a general connection condition is applied. All these types of interfaces are available in the software used for the simulations.

For the boundary conditions, the discharge velocity is set axial at the inlet of the computing domain with no tangential component. At the outlet, the pressure is set to the atmospheric pressure. No slip conditions are imposed for the velocity on the domain.

To compute the turbine performance the angular momentum balance equation is solved as presented in equation 10. Moreover, since numerical simulations do not include the mechanical losses, a calculation of the torque is performed to compare the transformed specific energy $E_{\mathrm{t}}$ calculated from the angular momentum budget with the one computed from the torque as follows:

$$
E_{\mathrm{t}}^{*}=\frac{P}{\rho \cdot Q_{\mathrm{t}}}=\frac{\omega_{\mathrm{A}} \cdot T_{\mathrm{A}}+\omega_{\mathrm{B}} \cdot T_{\mathrm{B}}}{\rho \cdot Q_{\mathrm{t}}}
$$

The transformed discharge is computed as $Q_{\mathrm{t}}=Q-Q_{\mathrm{r}, \mathrm{A}}-Q_{\mathrm{r}, \mathrm{B}}$. The discharge losses $Q_{r}$ in the tip radial clearance are computed by solving the following 
integral for each runner:

$$
Q_{\mathrm{r}}=\int_{A_{\mathrm{tip}}} \vec{C} \cdot \vec{n} \mathrm{~d} A_{\mathrm{tip}}
$$

where $A_{\text {tip }}$ is the area of the tip radial clearance.

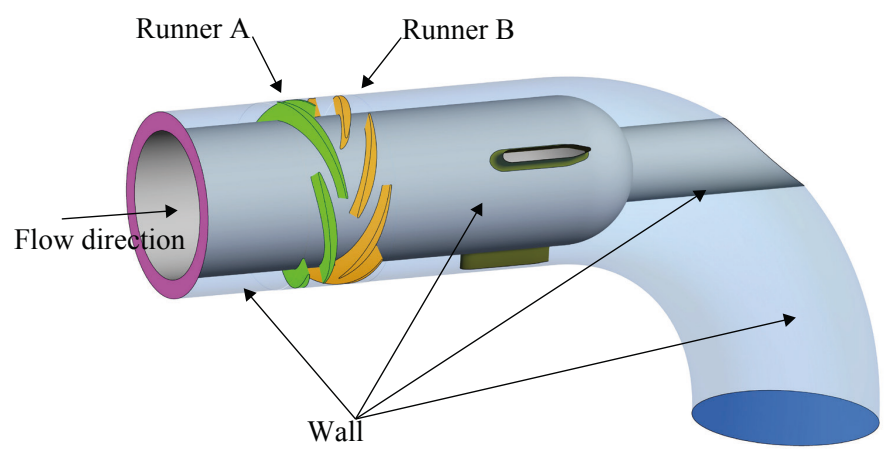

a)

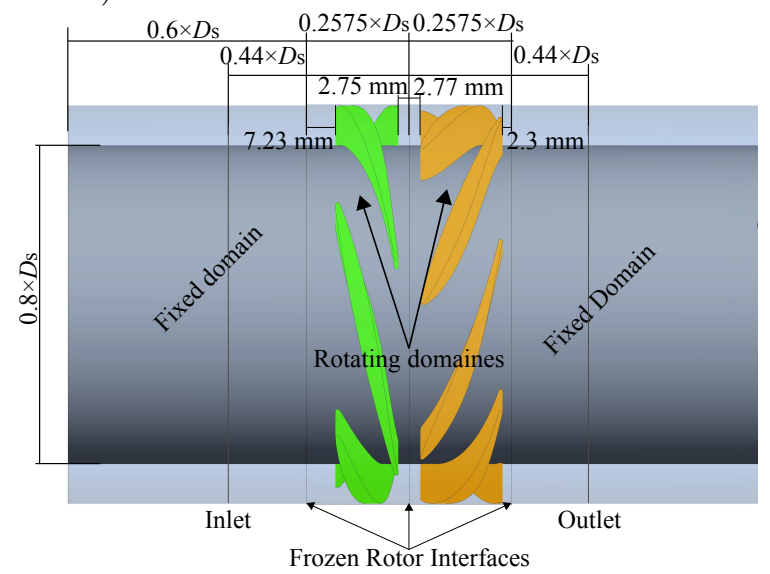

c)

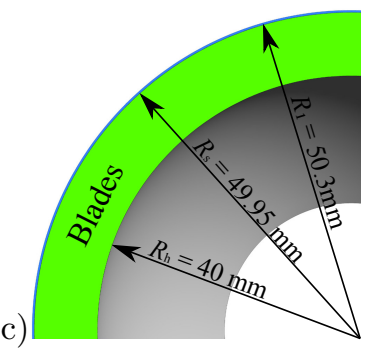

Fig. 5: Definition of the computing domain.

\subsection{Fulfillment of the velocity profiles}

Both the meridional and tangential velocity profiles measured by performing LDV must be completed in the span-wise since LDV measurements could not be achieved close to the hub wall of the micro-turbine due to the reflection of the laser beam. A first hypothesis on the velocity profile shape is done by taking the numerical simulations as a reference [20. By using third order 


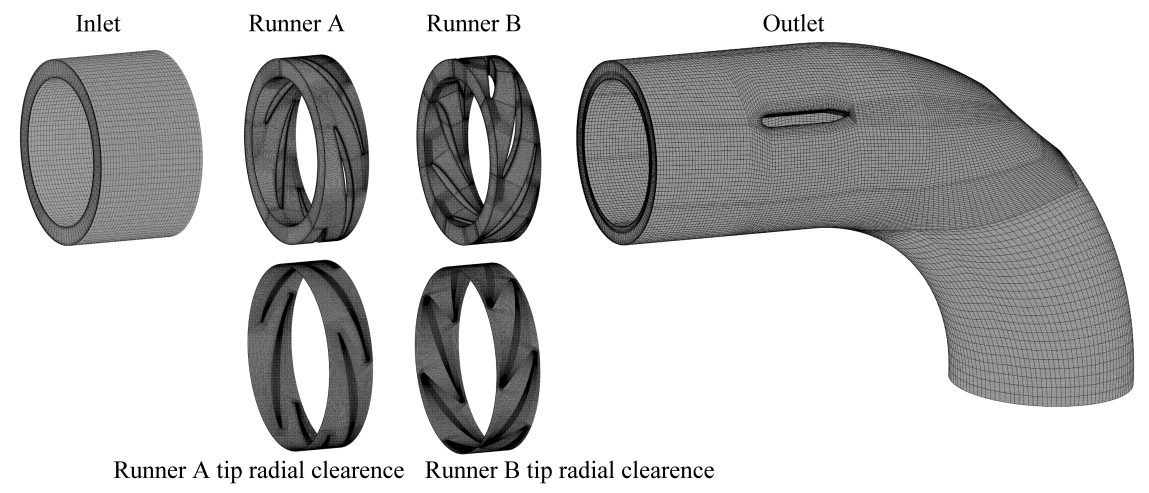

Fig. 6: Illustration of the meshes.

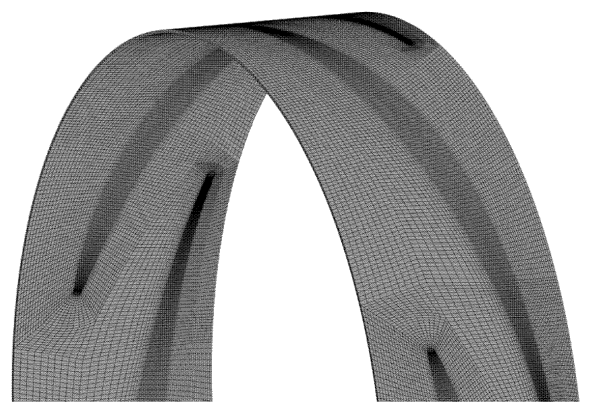

Runner A tip radial clearence

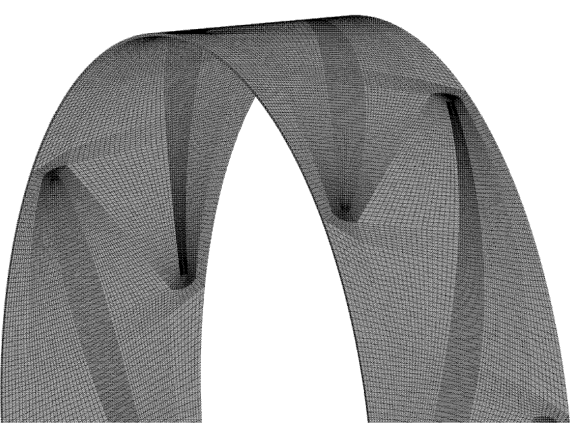

Runner B tip radial clearence

Fig. 7: Illustration of the meshes of the blade tip radial clearance.

Hermite polynomial 21], the interpolation of the measured average values of the meridional velocity component $C_{\mathrm{m}}$ is performed as a function of the radius $r$.

$$
C_{\mathrm{m}}(r)=\sum_{i=0}^{3} b_{i} H e_{i}(r)
$$

The mass balance equation, equation 14, is then integrated on the profiles to compute the discharge $Q_{\mathrm{t}}$ for each investigated section.

$$
Q_{\mathrm{t}}=\int_{A} \vec{C} \cdot \vec{n} \mathrm{~d} A=\int_{R_{\mathrm{h}}}^{R_{\mathrm{s}}} 2 \pi C_{\mathrm{m}}(r) r \mathrm{~d} r
$$

The discrepancies between the discharges computed in the three sections and the measured one $Q$ must be minimized. Thus an optimization problem is solved 
Tab. 2: Main characteristics of the meshes

\begin{tabular}{c|cccccc}
\multicolumn{1}{c}{} & $\begin{array}{c}\text { Number } \\
\text { of nodes }\end{array}$ & $\begin{array}{c}\text { Number } \\
\text { of elements }\end{array}$ & $y_{\min }^{+}$ & $y_{\max }^{+}$ & $y_{\text {mean }}^{+}$ & $\begin{array}{c}\text { Min } \\
\text { angle }\end{array}$ \\
\hline Inlet & $1^{\prime} 0^{\prime} 800$ & $94^{\prime} 656$ & 8.4 & 23.0 & 10.7 & 84.1 \\
Runner A & $1^{\prime} 031^{\prime} 125$ & $964^{\prime} 560$ & 1.0 & 56.1 & 14.2 & 52.5 \\
Runner A tip radial clearance & $241^{\prime} 540$ & $179^{\prime} 520$ & 5.7 & 665.1 & 52.0 & 46.6 \\
Runner B & $1^{\prime} 520^{\prime} 400$ & $1^{\prime} 426^{\prime} 320$ & 0.2 & 62.3 & 11.1 & 49.2 \\
Runner B tip radial clearance & $263^{\prime} 592$ & $196^{\prime} 287$ & 15.9 & 360.4 & 46.9 & 57.3 \\
Outlet & $798^{\prime} 406$ & $757^{\prime} 858$ & 1.1 & 121.7 & 31.7 & 30.1 \\
Total & $3^{\prime} 946^{\prime} 863$ & $3^{\prime} 619^{\prime} 201$ & & & & 30.1
\end{tabular}

whose objective function is defined by equation 15 and equation 16 .

$$
\begin{gathered}
g\left(b_{i}\right)=\left|\int_{R_{\mathrm{h}}}^{R_{\mathrm{s}}} 2 \pi C_{\mathrm{m}}(r) r \mathrm{~d} r-Q\right| \\
b_{i}=\arg \min \left(g\left(b_{i}\right)\right)
\end{gathered}
$$

The velocity values are bounded within the standard deviation $\sigma$ of the measured values as expressed in equation 17 .

$$
\bar{C}_{\mathrm{m}}-\sigma(r) \leq C_{\mathrm{m}}(r) \leq \bar{C}_{\mathrm{m}}+\sigma(r)
$$

Since measurements could not be achieved close to the hub, a constraint on the first order derivative is imposed to both velocity components for $0.8<r / R_{\mathrm{S}}<$ 0.86 to assure the decrease of the velocity amplitude owing to the wall effect as predicted by the numerical simulation.

$$
\frac{\mathrm{d} C_{\mathrm{m}}(r)}{\mathrm{d} r}>0 \quad \frac{\mathrm{d} C_{\mathrm{u}}(r)}{\mathrm{d} r}>0
$$

The absolute velocity and the relative flow angle are computed spanwise according to equation 19 and equation 20.

$$
\begin{gathered}
C(r)=\sqrt{C_{\mathrm{m}}(r)^{2}+C_{\mathrm{u}}(r)^{2}} \\
\beta(r)=\arctan \frac{C_{\mathrm{m}}(r)}{U(r)+\left|C_{\mathrm{u}}(r)\right|}
\end{gathered}
$$


Tab. 3: Summary of the investigated operating points (OP).

\begin{tabular}{c|cccccc} 
OP & $Q\left(\mathbf{m}^{3} / \mathbf{s}\right)$ & $E(\mathbf{J} / \mathbf{K g})$ & $N_{\mathrm{A}}\left(\mathbf{m i n}^{-1}\right)$ & $N_{\mathrm{B}}\left(\mathbf{m i n}^{-1}\right)$ & Symbol & Comment \\
\hline OP1 & $10.4 \cdot 10^{-3}$ & 251 & 2410 & 2410 & $\times$ & BEP $a=1$ \\
OP2 & $7.1 \cdot 10^{-3}$ & 125 & 1548 & 1858 & $\bullet$ & BEP1 on cam speed \\
OP3 & $10.4 \cdot 10^{-3}$ & 248 & 2398 & 2648 & $\circ$ & BEP2 on cam speed \\
OP4 & $8.7 \cdot 10^{-3}$ & 196 & 3000 & 3000 & $\triangle$ & Design Point
\end{tabular}

\subsection{Investigated operating conditions}

Steady state measurements and numerical simulations are performed for the operating condition summarized in Tab. 3. The hillchart of the machine is illustrated in Fig. 8 for the on-cam speed operating points and Fig. 9 for the operating points at $a=1$ (equation 21) 8]. During the experimental measurements, the rotational speed values of the runners are set through a controller and the discharge by controlling the pump rotation.

The relative rotational speed coefficient is defined as follows:

$$
a=\frac{\omega_{\mathrm{A}}}{\omega_{\mathrm{B}}}
$$

\section{Results}

4.1. Numerical investigation of blade tip radial clearance effect on the velocity profiles

Numerical simulations with and without the blade tip radial clearance between the runner and the duct are carried to assess the influence of this parameter on the turbine performances. In the turbine, there is a tip radial clearance of $0.35 \mathrm{~mm}$ representing $7 \%$ of the radius; the section of the tip radial clearance representing $3.9 \%$ of the full flow section. The streamlines are represented in Fig. 10in both computing domains for the same operating conditions. It is noticed that the fluid going through the tip radial clearance section modifies the flow at the runners sections and downstream. 


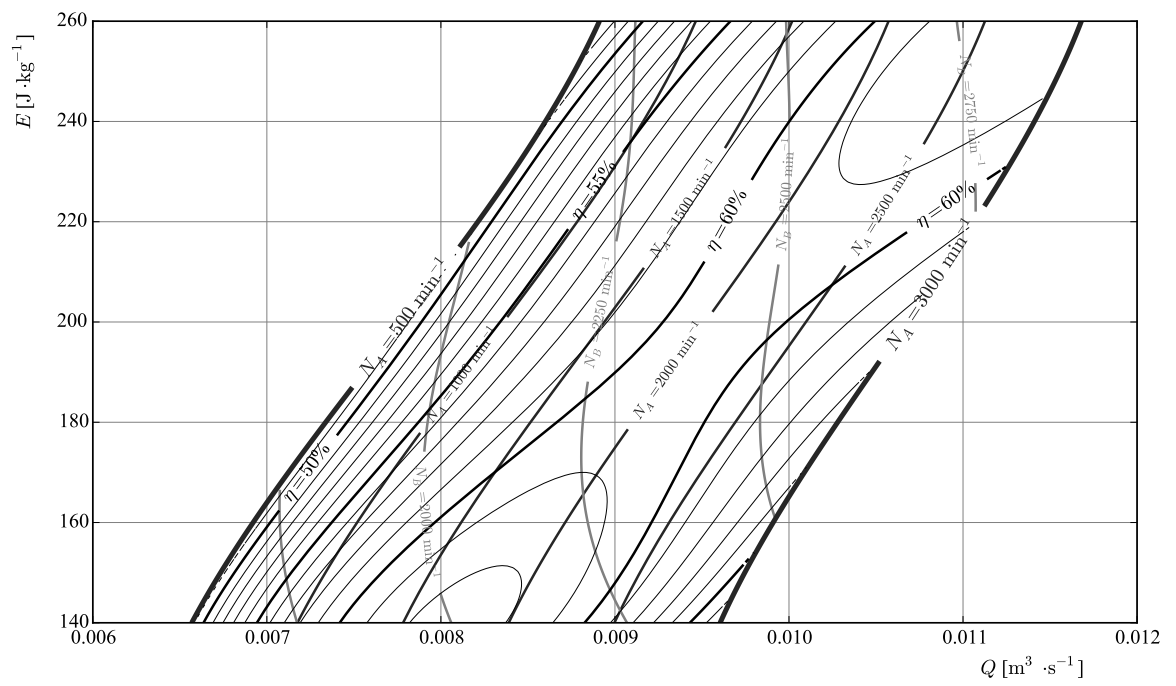

Fig. 8: Discharge-specific energy hillchart of the micro-turbine for on cam speed.

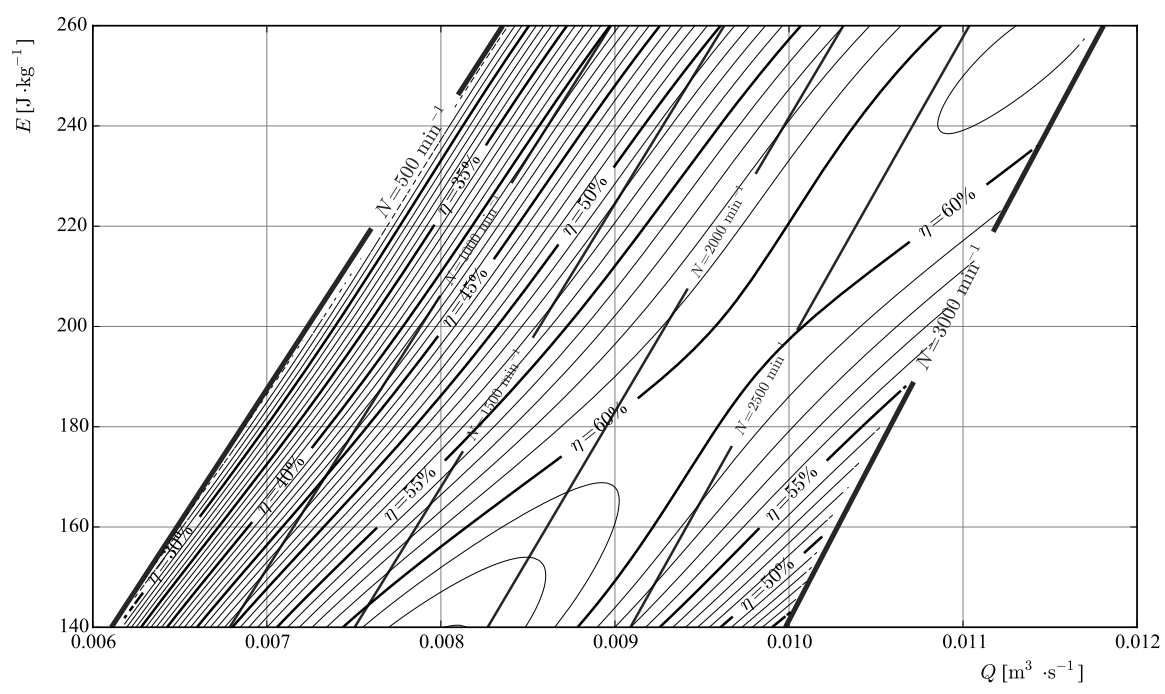

Fig. 9: Discharge-specific energy hillchart of the micro-turbine at identical rotating speed values, i.e. at $a=1$. 
In Fig. 11, the performance computed for the four investigated operating points are presented. The specific energy coefficient $\psi$ is computed as follows in equation 22

$$
\psi=\frac{2 E_{t}}{\left(\omega_{\mathrm{A}} R_{\mathrm{s}}\right)^{2}}
$$

This coefficient is computed for both cases with and without the tip radial clearance. In Fig. 11 $)$ it is observed that $\psi$ is influenced by the tip radial clearance: for a given operating point, the specific energy coefficient is higher in the simulation without the tip radial clearance than in the one with the tip radial clearance. This can be explained by the fact that the blockage effect is lower in the configuration with the tip radial clearance and this causes a decrease of the specific energy of turbine.

180 The energy losses factor $e_{\mathrm{r}}$, defined in equation 9 is computed by solving both the angular momentum balance equation, as presented in equation 10 and by solving the torque equation, as in equation 11. The results are presented in Fig. 11b). The two methods to calculate the transformed energy give similar results and confirm the approach used in the experimental measurements. The energy losses factor is lower in the configuration without the tip radial clearance compared to the one with the tip radial clearance since, as previously observed, specific energy of turbine decrease in the configuration with the tip radial clearance.

The discharge losses factor $q_{\mathrm{r}}$, defined in equation 9, is also presented in Fig. 11b). A higher discharge corresponds to a higher amount of fluid going through the tip radial clearance.

The hydraulic efficiency $\eta_{\mathrm{h}}$ defined in equation 7 and 8 and computed by using both momentum balance and torque equation, respectively, are presented in Fig. 11k). As for the calculation of the energy losses factor, the two methods give similar results. On the other hand, a difference on the configuration with/without tip radial clearance is observed: a lower efficiency is computed by the simulation with the tip radial clearance, coherently with the computation of higher losses factors. 


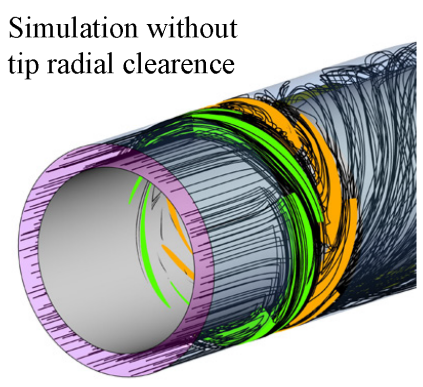

Simulation with tip radial clearence

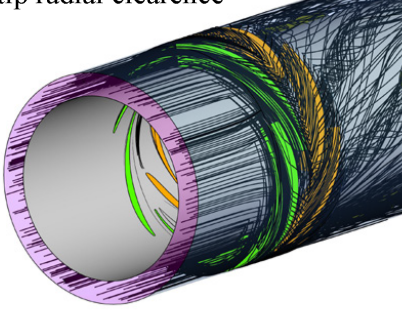

Fig. 10: Streamlines in the turbine without (left) and with the blade tip radial clearance (right).

\subsection{Velocity profiles and wall pressure at the inlet and outlet sections}

The meridional and tangential velocity profiles at the inlet section of the micro-turbine, obtained both experimentally and numerically, are illustrated in Fig. 12. Both velocity components are made dimensionless by the reference discharge velocity $C_{\text {ref }}=Q / A$.

$$
\begin{gathered}
c_{\mathrm{m}}=\frac{C_{\mathrm{m}}}{C_{\mathrm{ref}}} \\
c_{\mathrm{u}}=\frac{C_{\mathrm{u}}}{C_{\mathrm{ref}}}
\end{gathered}
$$

200 that the flow is axial for the investigated operating points at the inlet section of the first runner. In addition, it is observed that the flow is independent of the operating condition in this section of the machine.

The meridional and tangential velocity profiles at the outlet section of the 205 micro-turbine obtained both experimentally and numerically are illustrated in Fig. 13. A good agreement between experimental measurements and numerical simulations for both the velocity components is observed.

The absolute velocity profile and the relative flow angle are illustrated in Fig. 14. The absolute velocity component is made dimensionless by the reference discharge velocity:

$$
c=\frac{C}{C_{\mathrm{ref}}}
$$




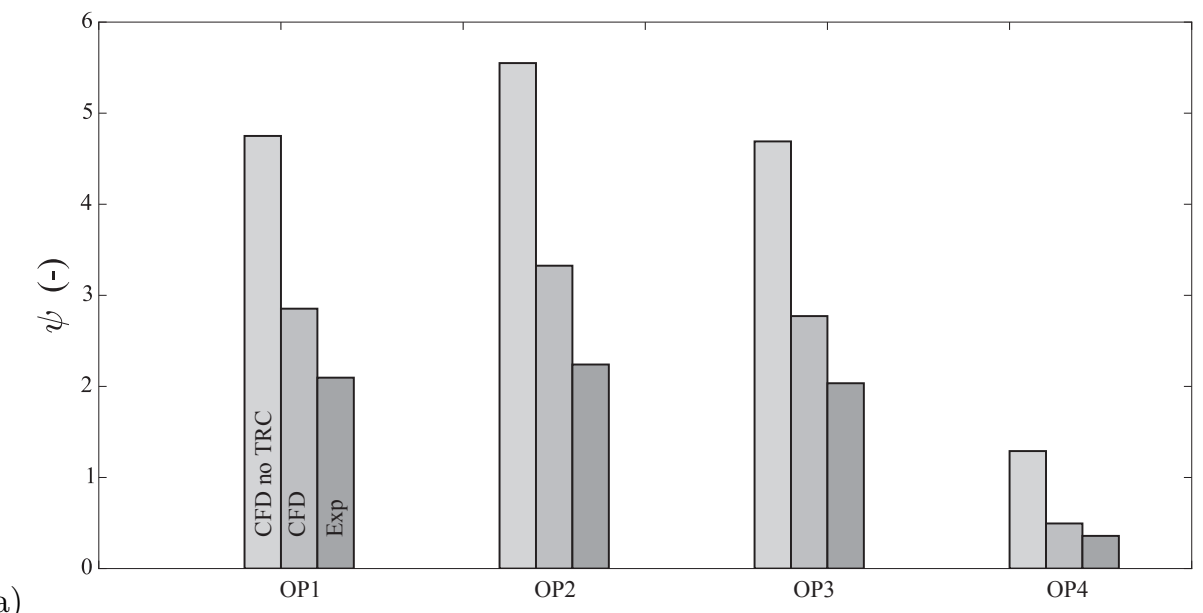

a)

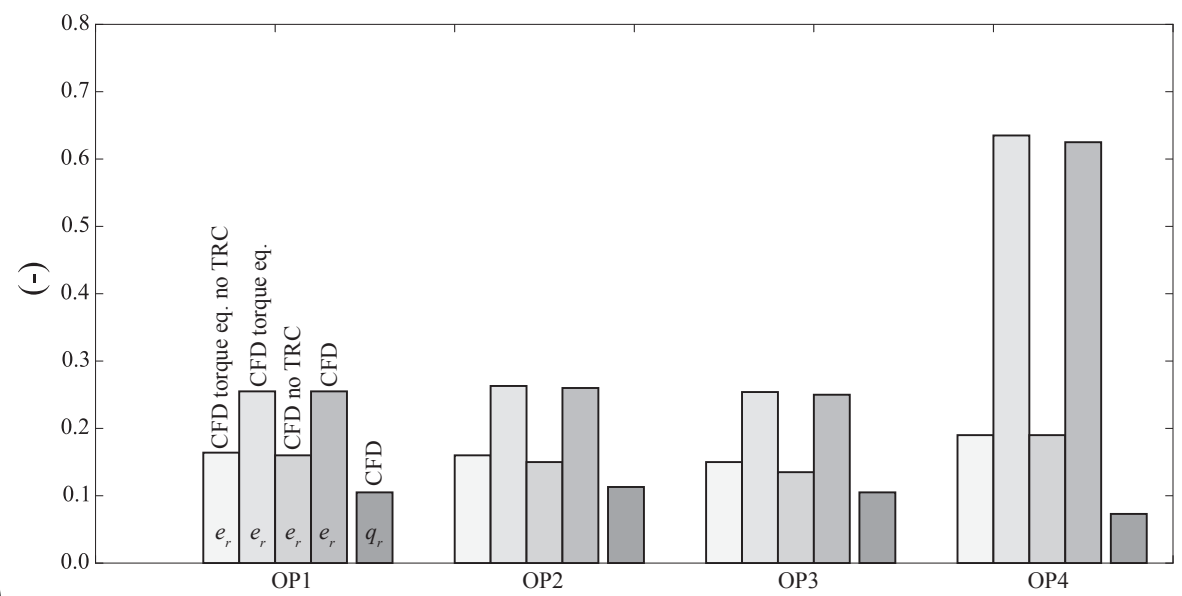

b)

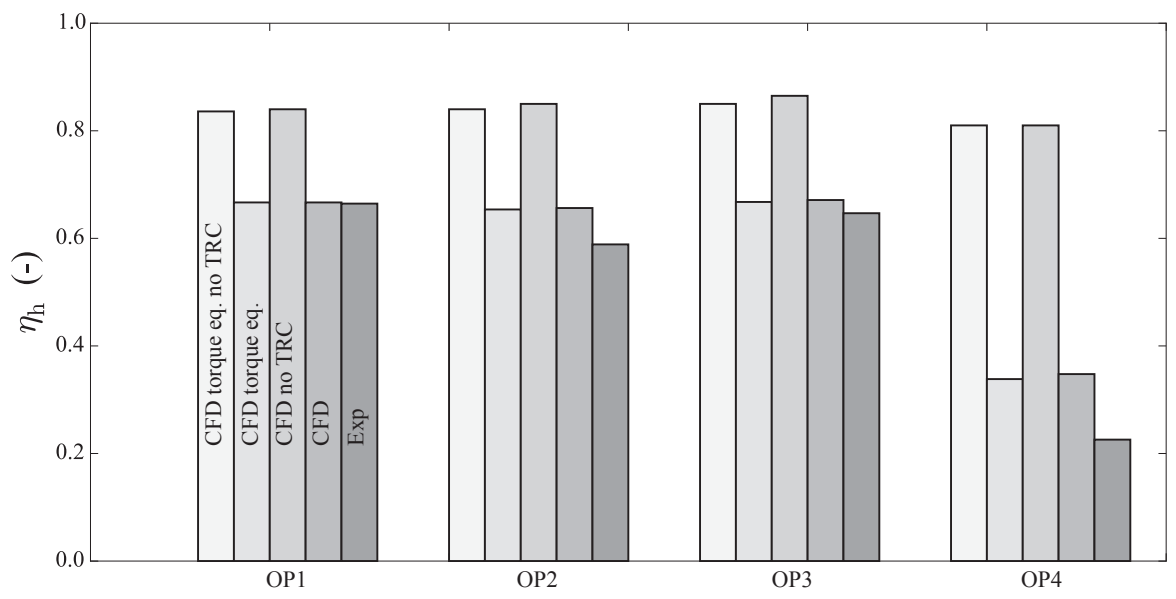

c)

Fig. 11: Performance of the micro-turbine computed by numerical simulations by solving the momentum balance (CFD) and the torque equation (CFD torque eq.) in the configuration with blade tip radial clearance and without (no TRC), and by experimental investigations (Exp). a) Specific energy coefficient. b) Energy and discharge losses factors. c) Hydraulic efficiency. 
It is observed that the flow follows the blade deviation at design point.
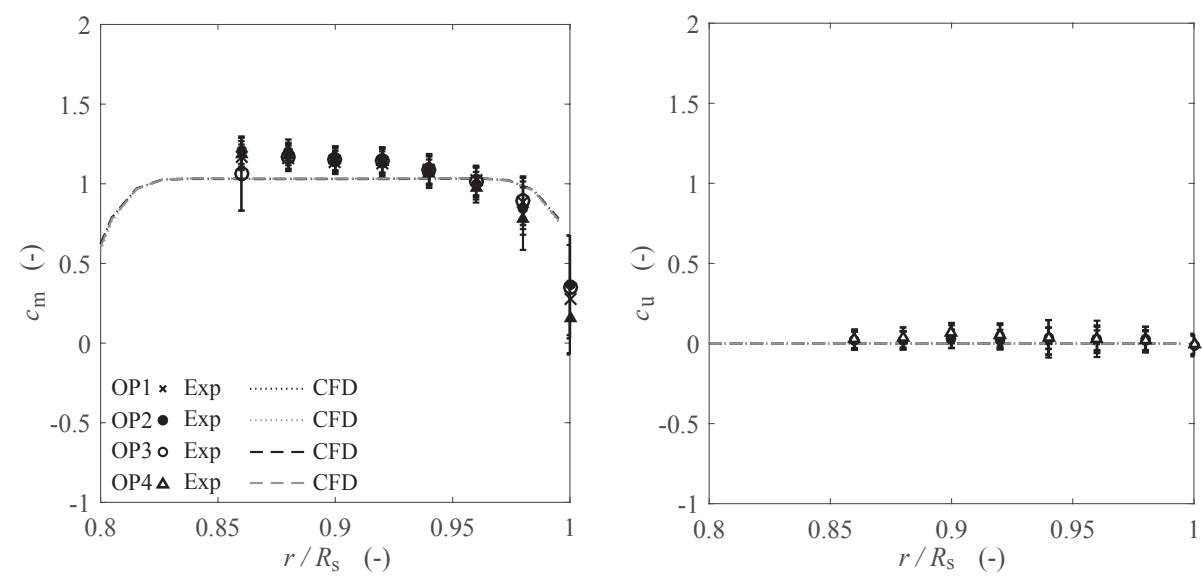

Fig. 12: Meridional (left) and tangential (right) velocity profiles at the inlet section of the micro-turbine with counter rotating runners.
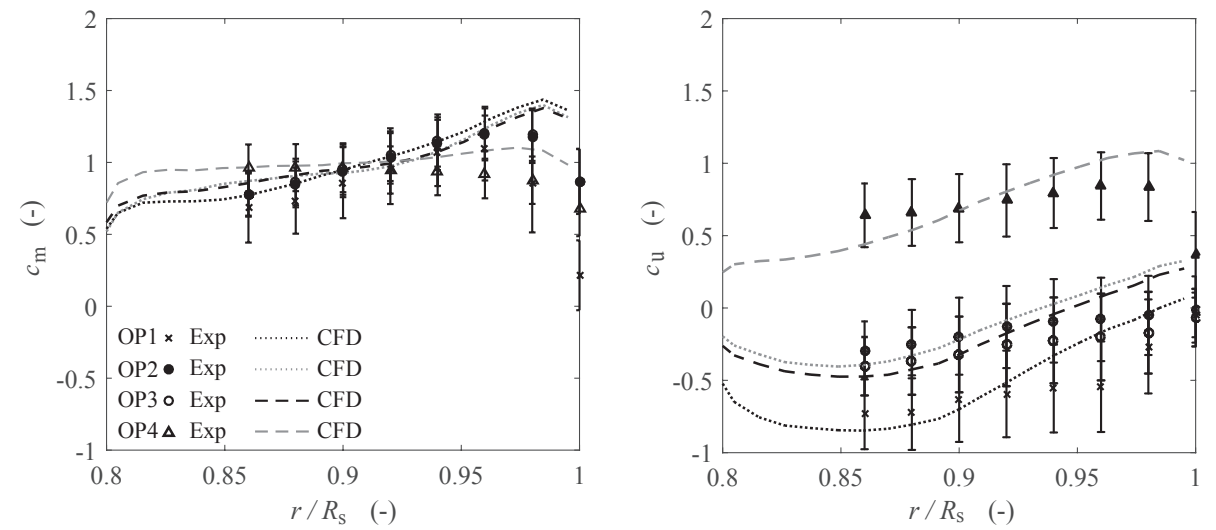

Fig. 13: Meridional (left) and tangential (right) velocity profiles at the outlet section of the micro-turbine with counter rotating runners.

\subsection{Performance of the machine}

The comparison of the energy coefficient and hydraulic efficiency computed by both numerical simulation and experimental measurements are presented in 

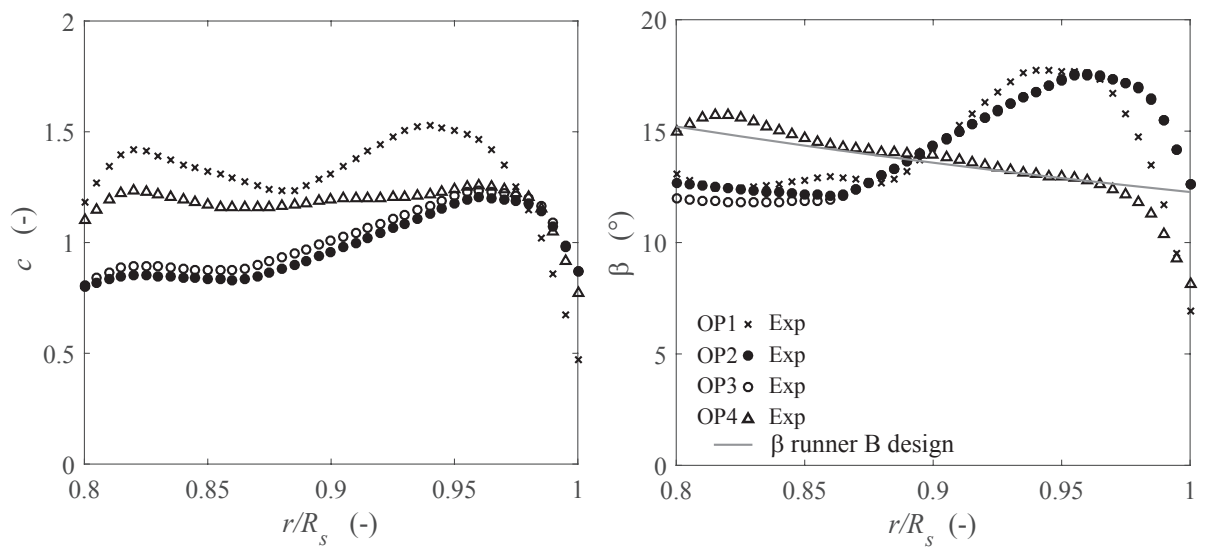

Fig. 14: Absolute velocity profile (left) and relative flow angle (right) at the outlet section of the micro-turbine with counter rotating runners.

Fig. 11. The overestimation of the energy coefficient and of the hydraulic efficiency is due to the limitation of the numerical simulation which does not take into account the anisotropy of the flow and flow leakage in the vaneless gap between the two runners.

\section{Conclusion}

Experimental measurements and numerical simulations are performed to investigate the flow characteristics and the hydraulic performance of a microturbine. A method based on the angular momentum balance is developed to compute the hydraulic performance.

A good agreement between the experimental measurements and the numerical computations are observed on the velocity profiles in the meridional and tangential components for both the inlet and outlet sections. An inlet axial flow and an outlet flow deviation by the blade angle are observed at the operating points for the best performance.

Limitation of the numerical method in predicting the flow condition in the section between the two runners causes an overestimation of the specific energy 
coefficient and of the efficiency. The best performance is observed for the operating points which feature a flow whose deviation at the outlet section does not follow the blade angle. This means that further improvements on the blade shape of the runner can be done to achieve better performance of the machine.

The method presented in this paper provides a solution to compute the 235 hydraulic performance of a hydraulic turbine. The measurement of the supply power is reduced by the mechanical losses which have a higher impact on the efficiency of a small hydro-turbine than a conventional turbine. The presented method can be helpful in the investigation of the hydraulic performance of a small hydro-turbine, where the measurements are difficult to achieve due to the limited available space in the machine.

\section{Acknowledgments}

The research leading to the results published in this paper has received funding from SCCER SoE, the Swiss Energy Center for Energy Research Supply of Electricity granted by the Swiss Commission for Technology and Innovation

24 (CTI); the Ark, the foundation for innovation of Valais Canton, through the Project HydroVS and from the Swiss Commission for Technology and Innovation as part of the DuoTurbo project number 17197.1 PFEN IW. The authors would like to thank the hydroelectricity team of the HES SO Valais for their collaboration and technical support. Moreover, the authors would like to acknowledge the technical staff of the Laboratory for Hydraulic Machines, especially Georges Crittin, David Buzzi, Christian Sierro and Vincent Berruex.

\section{References}

[1] Statistics of hydroelectric installations in switzerland (2016). URL http://www . bfe .admin . ch/themen/00490/00491/?lang=en

255 [2] I. Samora, V. Hasmatuchi, C. Münch-Alligné, M. Franca, A. Schleiss, H. Ramos, Experimental characterization of a five blade tubular propeller turbine for pipe inline installation, Renewable Energy 95 (2016) 356 - 366. 
[3] N. Lee, J. Choi, Y. Hwang, Y. Kim, Y. Lee, Performance analysis of a counter-rotating tubular type micro-turbine by experiment and cfd, proceedings of 26th IAHR Symposium on Hydraulic Machinery and Systems published in IOP Conference Series: Earth and Environmental Science, 15 (2012). doi:10.1088/1755-1315/15/4/042025

[4] A. McNabola, P. Coughlan, L. Corcoran, C. Power, A. Williams, I. Harris, J. Gallagher, D. Styles, Energy recovery in the water industry using microhydropower: an opportunity to improve sustainability, Water policy (2014) 168-183doi:http://dx.doi.org/10.2166/wp.2013.164.

[5] P. Wiemann, G. Müller, Senior risk management and resolution strategies for established and novel technologies in the low head small hydropower market hidroenergia, Ljubljana.

[6] L. Ferro, L. Gato, A. Falcão, Design of the rotor blades of a mini hydraulic bulb-turbine, Renewable Energy 36 - 9 (2011) 2395 - 2403.

[7] D. Biner, V. Hasmatuchi, D. Violante, S. Richard, S. Chevailler, L. Andolfatto, F. Avellan, C. Münch-Alligné, Engineering and performance of duoturbo: Microturbine with counter-rotating runners, proceedings of 28th IAHR Symposium on Hydraulic Machinery and Systems published in IOP Conference Series: Earth and Environmental Science 49 (2016). doi:10.1088/1755-1315/49/10/102013.

[8] L. Andolfatto, J. Delgado, E. Vagnoni, C. Münch-Alligné, F. Avellan, Analytical hillchard towards the maximisation of energy recovery on water utility networks with counter rotating micro-turbine, E-proceedings of 36th IAHR World Congress, The Hague, The Netherland, June 2015.

[9] L. Andolfatto, C. Euzenat, E. Vagnoni, C. Münch-Alligné, F. Avellan, A mixed standard/custom design strategy to minimize cost and maximize efficiency for picohydro power potential harvesting, E-proceedings of 5th 285 International Youth Conference on Energy (IYCE), Pisa, May 2015doi: 10.1109/IYCE.2015.7180729 
[10] J. Gagnon, M. Iliescu, G. Ciocan, C. Deschnes, Experimental investigation of runner outlet flow in axial turbine with $l d v$ and stereoscopic piv, E-proceedings of 24th IAHR Symposium on Hydraulic Machinery and Systems, Foz do Iguassu, October 2008.

[11] S. Zaidi, Practical problems associated with laser anemometry in high speed turbomachines, Optics and Lasers in Engineering 26 (1998) 473-486. doi: 0143-8166 (95) 00126-3.

[12] R. Miorini, H. Wu, J. Katz, The internal structure of the tip leakage vortex within the rotor of an axial waterjet pump, J. Turbomachines. 134(3) (2011) 031018 .

[13] A. Müller, M. Dreyer, N. Andreini, F. Avellan, Draft tube discharge fluctuations during self-sustained pressure surge: fluorescent particle image velocimetry in two-phase flow, Experiments in Fluids 54 issue 4 (2013) $1-11$.

[14] A. Müller, A. Favrel, C. Landry, Y. Yamamoto, F. Avellan, On the physical mechanisms governing self-excited pressure surge in francis turbines, proceedings of 27th IAHR Symposium on Hydraulic Machinery and Systems published in IOP Conference Series: Earth and Environmental Science 22 issue 3 (2014). doi:10.1088/1755-1315/22/3/032034.

[15] O. Uzol, Y. C. Chow, J. Katz, C. Meneveau, Unobstructed piv measurements within an axial turbo-pump using liquid and blades with matched refractive indices, Experiments in Fluids 33 (2002) 909-919.

[16] Z. Zhang, LDA application methods, Springer, 2010.

[17] F. Menter, Two-equation eddy-viscosity turbulence models for engineering application, AIAA Journal 32(8) (1994) 1598-1605.

[18] D. Wilcox, Comparison of two-equation turbulence models for boundary layers with pressure gradient, AIAA Journal 31(8) (1993) 1414-1421. 
[19] B. Launder, D. Spalding, The numerical computation of turbulent flows, Computer Methods in Applied Mechanics and Engineering 3(2) (1974) 269289.

[20] C. Münch-Alligné, S. Richard, B. Meier, V. Hasmatuchi, F. Avellan, Numerical simulations of a counter-rotating micro-turbine., Advances in $\mathrm{Hy}-$ droinformatics, P. Gourbesville et al. (eds.), Springer Hydrogeology (2014) $363-373$ doi:10.1007/978-981-4451-42-030

[21] M. Abramowitz, I. Stegun, Handbook of Mathematical Functions, Dover, 1964. 